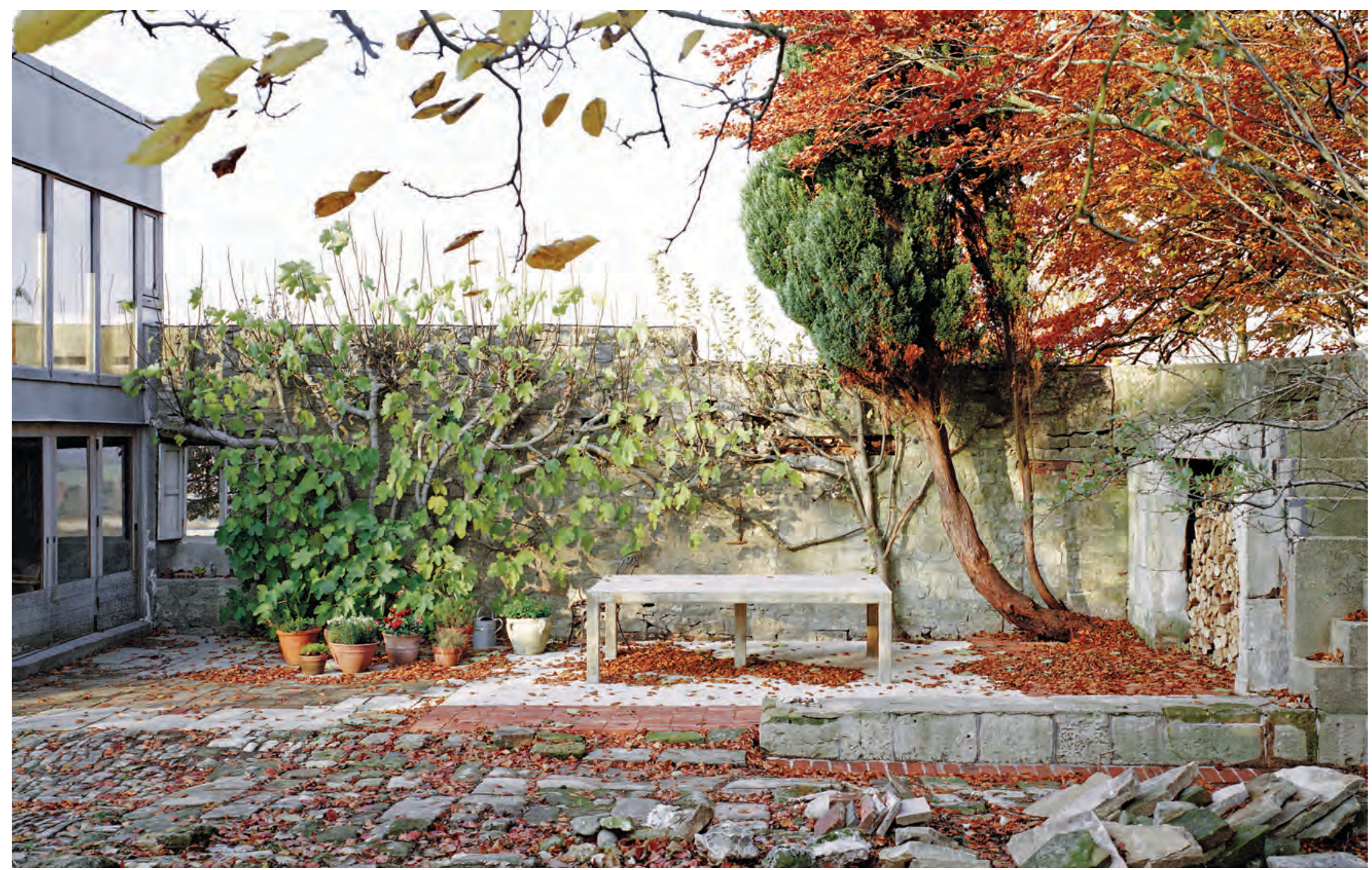

Jonathan Sergison | Profesor, Accademia di Architettura di Mendrisio.

Arquitecto, Architectural Association, 1989. Entre 1989 y 1995 colaboró sucesivamente en los estudios de David Chipperfield y Tony Fretton en Londres. En 1996 fundó, junto a Stephen Bates, Sergison Bates architects. Fue profesor en diversas universidades inglesas como University of Nottingham, University of North London, Architectural Association y University of Bath; fue profesor invitado en el departamento de arquitectura de ETH en Zürich y en École Polytechnique Fédérale en Lausanne.

Stephen Bates | Profesor, Technische Universität München.

Arquitecto, Royal College of Art, 1989. Antes de fundar Sergison Bates architects, colaboró con Liebman Villavecchia en Barcelona y con Bennetts Associates en Londres. Fue profesor en South Bank University, Architectural Association y University of Bath; fue profesor invitado en el Departamento de Arquitectura de ETH en Zürich y en École Polytechnique Fédérale en Lausanne.

Un delicado trabajo de redibujo y desmontaje constructivo establece una comprensión de las decisiones originales de Alison y Peter Smithson. El resultado incluye un set de meticulosas planimetrías, base para definir nuevas operaciones. Palabras clave: Arquitectura - Inglaterra, restauración, Smithsons, Solar pavilion.

A thoughtful survey and a series of constructive dissections aims to understand the decision-making process carried out by the Smithsons. The outcomes include a set of thorough drawings that helped to delimit a new intervention.

Keywords: Architecture - England, restoring, Smithsons, Solar pavilion.

\section{Bibliografía sugerida}

LICHTENSTEIN, Claude y Thomas SCHREGENBERGER (eds.). As found: The discovery of the ordinary. Lars Müller, Baden, 2001.

PEZO, Mauricio y Sofía VON ELLRICHSHAUSEN

(curadores). META. Diez pabellones para Chile. Ediciones ARQ, Santiago, 2011.

PUENTE, Moisés y Anna PUYUELO (eds.). 2 G No 34 Sergison Bates. Editorial Gustavo Gili, Barcelona, 2005.

SMITHSON, Alison. "Tisbury” en BAKER, Jeremy (ed.). "A Smithson file". Architectural Association Journal, The Architectural Association, Londres, 1966.

Otras fotografías de la restauración de Upper Lawn están disponibles para los suscriptores ARQ en edicionesarq.c

\title{
PLANOS PARA LA RESTAURACIÓN DE UPPER LAWN SERGISON BATES ARCHITECTS
}




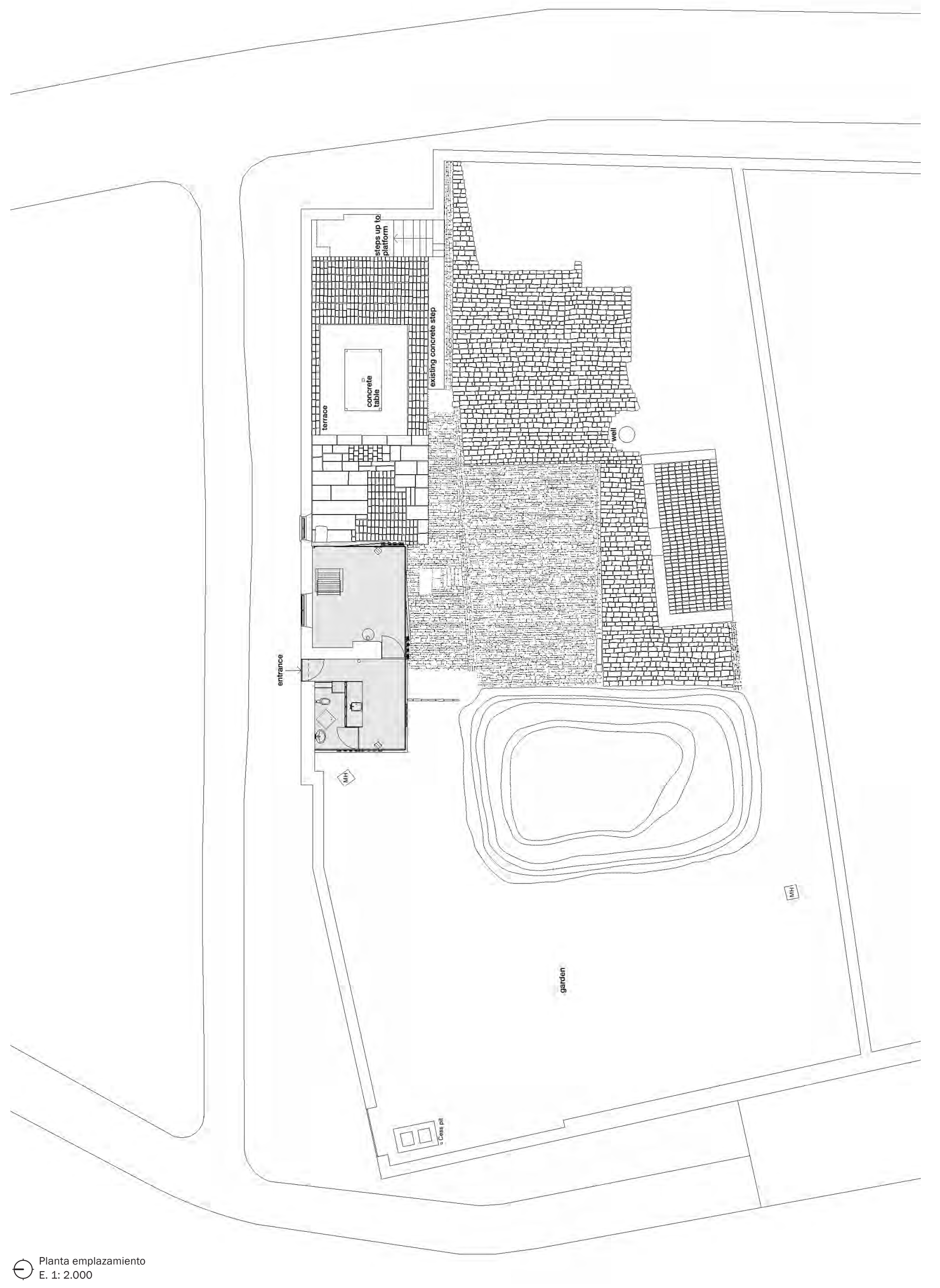

OBRAS Y PROYECTOS | WORKS AND PROJECTS 23 

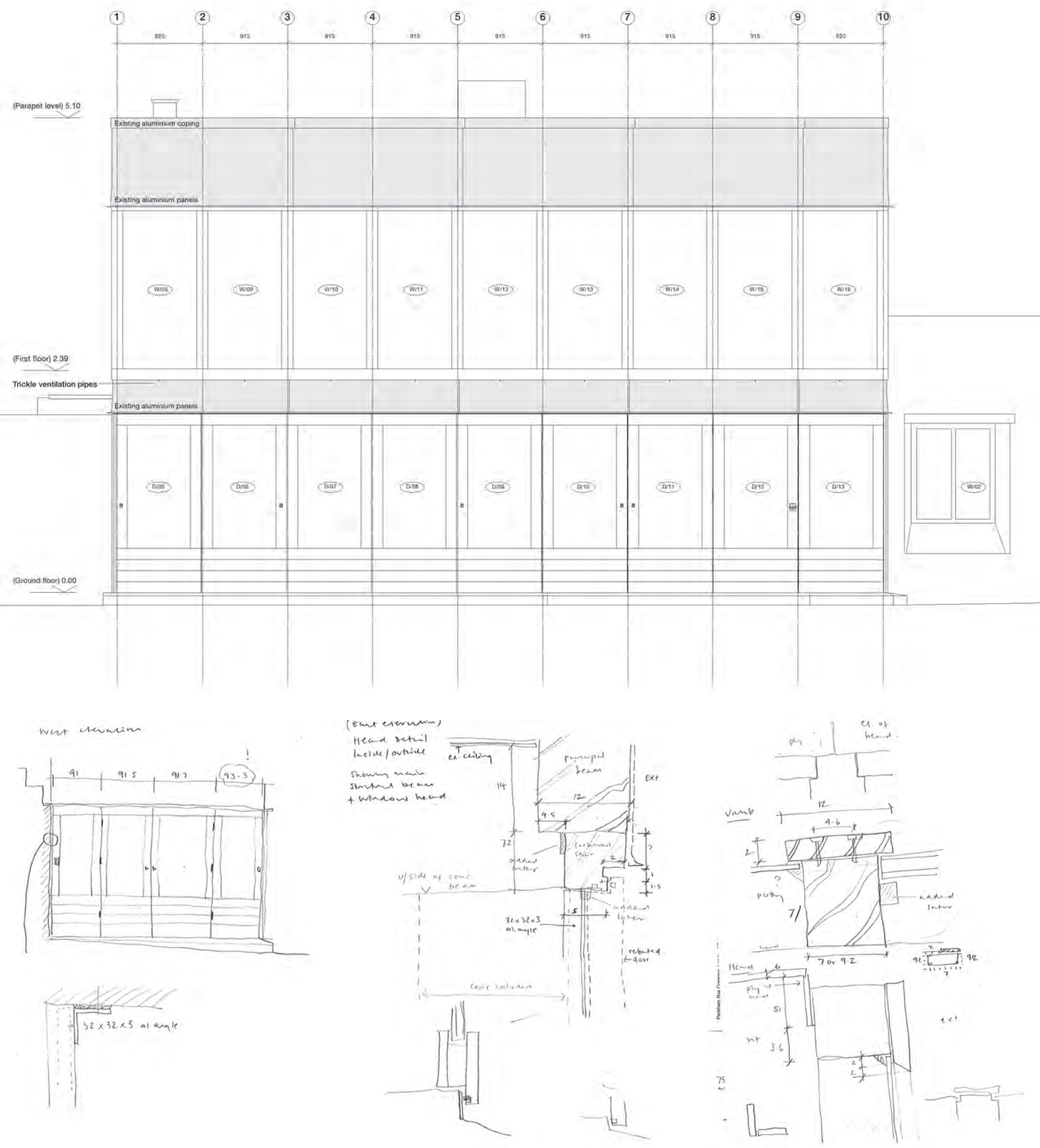

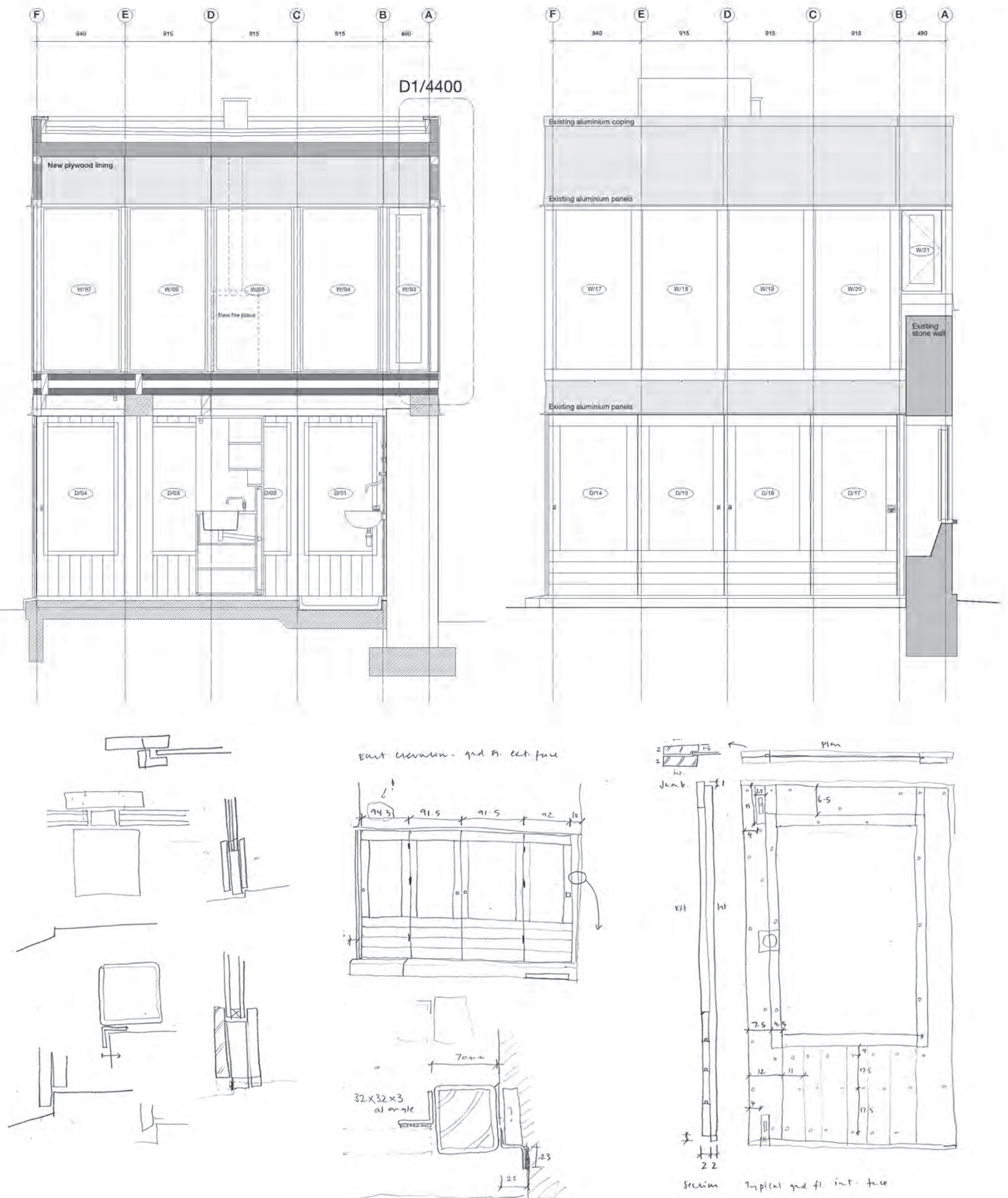


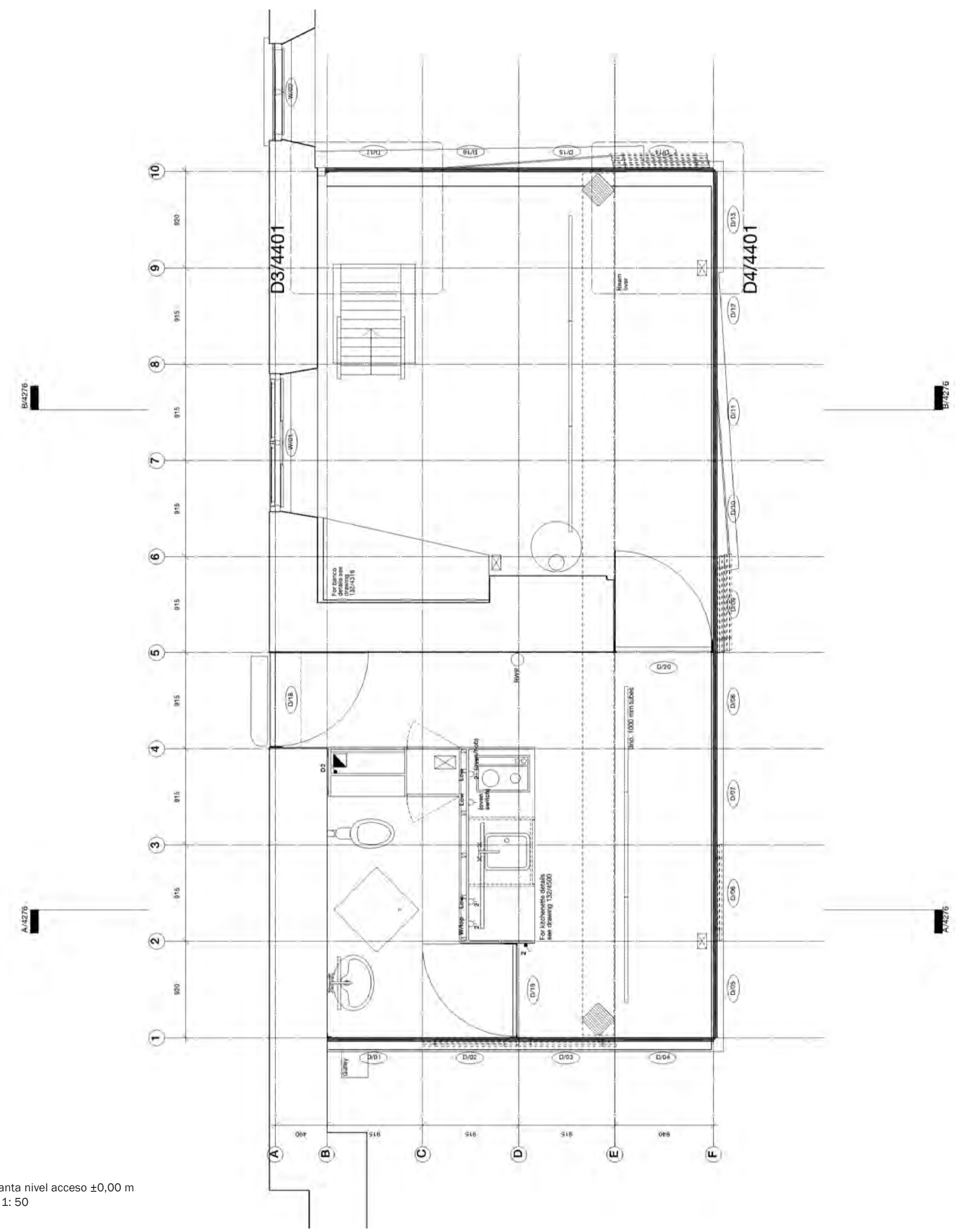

La restauración de la casa de fin de semana de Alison y Peter Smithson, emprendida por encargo de sus nuevos propietarios, supuso la realización de un exhaustivo levantamiento planimétrico y comprendió dos tipos de intervenciones. Por una parte, implementó pequeñas modificaciones que se sumaron a aquellas que los propios Smithson hicieran a la construcción tras usarla por casi dos décadas. Por otra, supuso el reemplazo discreto de algunas piezas y materiales desgastados por el tiempo y el acondicionamiento de las instalaciones para soportar los períodos más fríos del año.

También conocida como "Solar pavilion", la casa resulta de la articulación de una estructura ligera nueva y los restos de una construcción del s. XVIII que incluye un muro de piedra que cierra completamente el jardín de la propiedad. Las dos plantas suponen un esquema de ocupación informal y versátil, donde incluso el primer piso puede integrarse completamente al jardín por el repliegue de su cerramiento vidriado. 


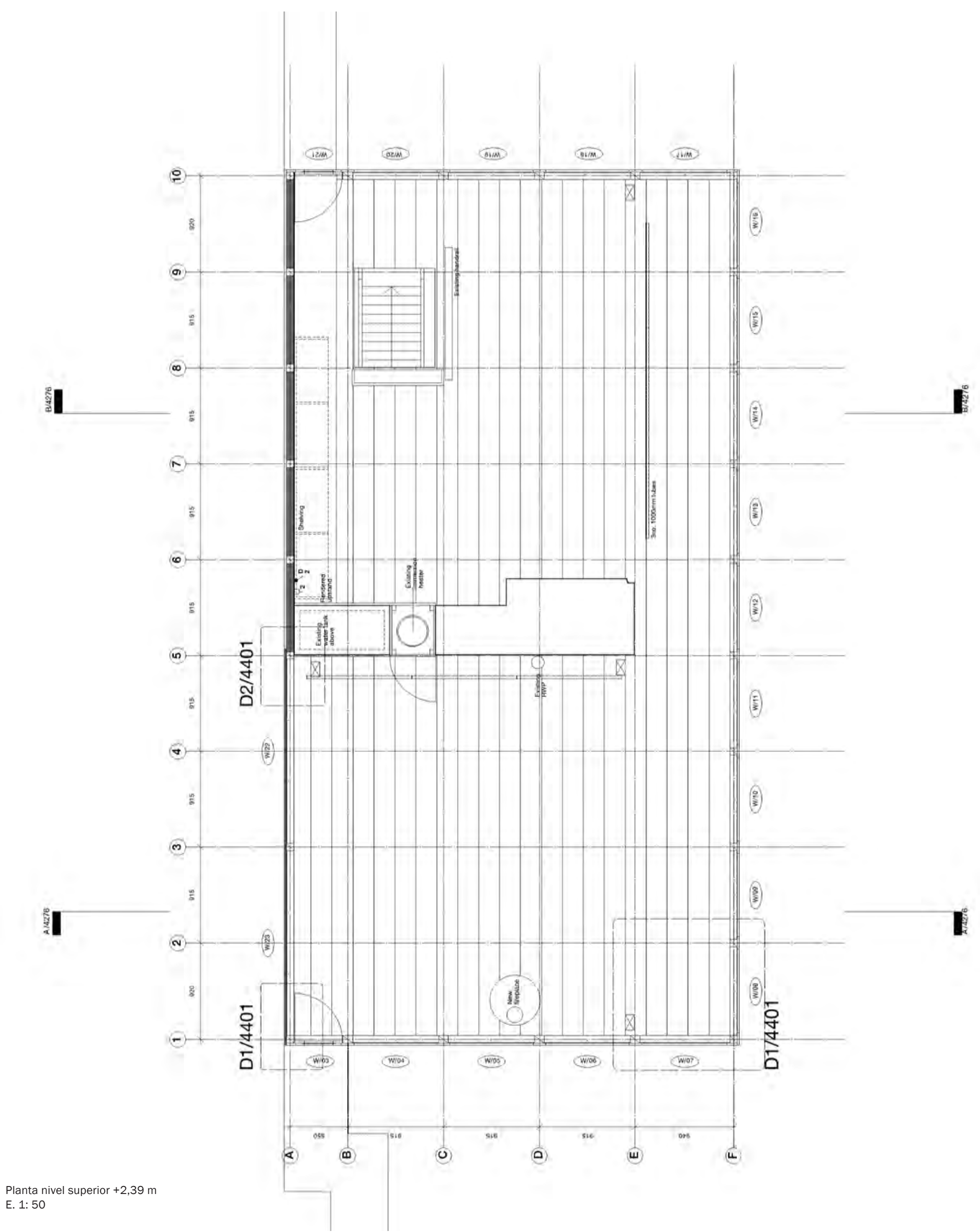

Probablemente la intervención más visible de Sergison Bates fue la construcción -en la terraza, junto a una chimenea exterior en el muro de piedrade una mesa de 2,5 x 1,5 m con cinco patas sobre una plataforma, en hormigón y armadura de acero inoxidable. La estructura propone otro régimen de uso para el exterior, en combinación con una serie de muebles plegables, y anticipa las posibilidades que un nuevo pabellón de una planta podría abrir al conjunto (PMH). ARQ
RESTAURACIÓN DE UPPER LAWN | Sergison Bates architects, 2004. Proyecto original de Alison y Peter Smithson. Tisbury, Inglaterra, 1962. | Restauración de $62 \mathrm{~m}^{2}$, más obras exteriores. | Mandantes: Ian y Jo Cartlidge. | Planos: Sergison Bates architects. | Fotografía: Ian Cartlidge. 